\title{
IL17A and IL17F genes polymorphisms are associated with histopathological changes in transplanted kidney
}

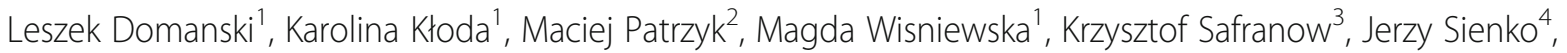
Tadeusz Sulikowski ${ }^{4}$, Marzena Staniszewska ${ }^{5}$ and Andrzej Pawlik ${ }^{5^{*}}$

\begin{abstract}
Background: Interleukin 17 is a proinflammatory cytokine involved in immune response after allograft transplantation. IL-17 family of proinflammatory cytokines includes IL-17A and IL-17F. Previous studies have demonstrated that the rs2275913 IL17A and the rs11465553 IL17F gene polymorphism are associated with kidney allograft function. Because of the association between these polymorphisms and post-transplant immune response, we assume that these single nucleotide polymorphisms may affect morphological structure of transplanted kidney.

The aim of this study was to examine the association of rs2275913 IL17A and rs2397084, rs11465553 and rs763780 IL17F gene polymorphisms with histopathological changes in transplanted kidney biopsies such as: glomerulitis, tubulitis, arteritis, cell infilitration and fibrosis.
\end{abstract}

Methods: The study enrolled 82 patients after renal graft transplantation in whom a kidney biopsy was performed because of impaired graft function. The rs2397084 T>C (Glu126Gly), rs11465553 G > A (Val155lle) and rs763780 T > C (His167Arg) polymorphisms within the IL17F gene and the rs2275913 A > G (- 197 A > G) polymorphism within the IL17A gene promoter were genotyped using TaqMan genotyping assays on a 7500 FAST Real-Time PCR System (Applied Biosystems, USA).

Results: There was a significant association between the rs2275913 IL17A gene polymorphism and the grade of tubulitis, which was more severe among patients with the A allele, compared to recipients with the GG genotype ( $G G$ vs. $A G+A A, P=0.02$ ), and with the grade of arteriolar hyaline thickening and mesangial matrix increase, which were more severe among patients with the $G$ allele compared to recipients with the AA genotype (AA vs. $\mathrm{AG}+\mathrm{GG}, P=0.01$ and $P=0.04$, respectively). Tubular atrophy and interstitial fibrosis were more severe among individuals with the $C$ allele at the rs763780 IL $17 F$ gene polymorphism (TT VS. TC, $P=0.09$ and $P=0.017$, respectively). However, it should be taken into account that the statistical significance was achieved without correction for multiple testing, and no significant association would remain significant after such correction.

Conclusions: The results of this study may suggest a possible association between the rs2275913 IL17A and rs2275913 IL17A gene polymorphisms and some histopathological changes in transplanted kidney biopsies.

Keywords: Cytokines, Histopathology, IL-17, Kidney allograft, Polymorphism

\footnotetext{
* Correspondence: pawand@poczta.onet.pl

${ }^{5}$ Department of Physiology, Pomeranian Medical University in Szczecin,

Powstancow Wlkp. 72, 70-111 Szczecin, Poland

Full list of author information is available at the end of the article
}

(c) The Author(s). 2019 Open Access This article is distributed under the terms of the Creative Commons Attribution 4.0 International License (http://creativecommons.org/licenses/by/4.0/), which permits unrestricted use, distribution, and reproduction in any medium, provided you give appropriate credit to the original author(s) and the source, provide a link to the Creative Commons license, and indicate if changes were made. The Creative Commons Public Domain Dedication waiver (http://creativecommons.org/publicdomain/zero/1.0/) applies to the data made available in this article, unless otherwise stated. 


\section{Background}

Interleukin 17 (IL-17) is a member of the IL-17 family of cytokines, which includes among others IL-17A and IL-17F. [1]. The complex structure of IL-17 and its pleiotropic function are still being investigated [2]. It was suggested that this cytokine forms a link between the innate and adaptive immune response [3]. Induction with IL-23 leads to enhanced IL-17 secretion by Th17, $\mathrm{CD}^{+} \mathrm{T}$ cells and $\gamma \delta$ T cells [4]. The biological activity of IL-17 is associated with proinflammatory actions in allergies, autoimmune diseases and cancer [3], moreover, it is also an important mediator in bacterial infections. However, recent studies revealed that IL-17 may also possess inhibitory functions and suppress the inflammatory response $[3,5,6]$. Numerous reports have confirmed that cytokine synthesis has a genetic background [7]. Genes encoding IL-17A and IL-17F are located on chromosome 6 (6p12) [8], and polymorphisms in these genes were studied in various autoimmune diseases [4, 9]. Studies regarding bone marrow and solid organ transplantation have highlighted the importance of $I L 17 A$ and IL17F gene polymorphisms in the pathogenesis of rejection; however, the nature of the posttransplant immune response is complex and differs between the early and late phase. The association between IL17A gene polymorphisms and acute rejection (AR) of kidney allografts is well known $[10,11]$, and the use of IL-17 concentrations as an early marker of AR has been considered [12]. However, there are only a few reports linking this cytokine with chronic rejection, and studies analysing kidney allograft histopathological changes are lacking [13]. Previous studies have demonstrated that the $\mathrm{G}$ allele of the rs2275913 IL17A gene polymorphism is associated with significantly impaired long-term kidney allograft function and that the GA genotype of the rs11465553 IL17F gene polymorphism is associated with a significantly higher risk of a return to dialysis after kidney transplantation [14]. Because of the association between analyzed polymorphisms and post-transplant immune response, we assume that these single nucleotide polymorphisms (SNPs) may affect morphological structure of transplanted kidney through presence of glomerulitis, tubulitis, arteritis, cell infilitration and fibrosis.

The aim of this study was to examine the association of rs2275913IL17A and rs2397084, rs11465553 and rs763780 IL17F gene polymorphisms with histopathological changes in transplanted kidney biopsies such as: glomerulitis, tubulitis, arteritis, cell infilitration and fibrosis.

\section{Methods}

The study enrolled 82 consecutive Caucasian patients after renal allograft transplantation (48 males and 34 females, mean age: $47.63 \pm 12.96$ years) in whom a kidney biopsy was performed between 2000 and 2006 because of impaired graft function, 183 kidney transplant recipients in whom no kidney biopsy was performed and 168 healthy controls. All biopsies were reviewed by a renal pathologist, and the Banff working classification criteria were used. The recipient's age and gender, the delayed graft function (DGF; which was defined as the need for dialysis during the first 7 days after transplantation), the AR occurrence and the number of episodes diagnosed clinically and confirmed by biopsy, chronic allograft nephropathy and chronic allograft dysfunction (CAD) were recorded in each case. All kidney allograft recipients were treated with the calcineurin inhibitor (cyclosporine A or tacrolimus), mycophenolate mofetil and glucocorticosteroids. The protocols differed between the patients because of changes in immunosuppressive therapies during the last 15 years. Additional patient characteristics are presented in Table 1. Blood samples for genetic analysis were collected from all patients at the start of the study, and informed consent was obtained from all patients. The local ethics committee at the Pomeranian Medical University, Szczecin, Poland, approved the study protocol (BN 120/2000) and written consent was obtained form all patients.

\section{Genotyping}

Genomic DNA was extracted from leukocytes contained in $200 \mu \mathrm{L}$ whole blood samples using a GeneMATRIX Quick Blood DNA Purification Kit (EURx, Poland). A DNA concentration was approximately $300 \mathrm{ng} / \mu \mathrm{l}$. The DNA was standardised using a Nanodrop ND-1000 spectrophotometer, and concentrations were equalised to $20 \mathrm{ng} / \mu \mathrm{l}$. This material was used as the matrix for amplification in a real-time polymerase chain reaction (RT-PCR).

The rs2397084 T>C (Glu126Gly), rs11465553 G> A (Val155Ile) and rs763780 $\mathrm{T}>\mathrm{C}$ (His167Arg) polymorphisms within the $I L 17 F$ gene and the rs2275913 A > G (-197 A > G) polymorphism within the IL17A gene promoter were genotyped using TaqMan genotyping assays on a 7500 FAST Real-Time PCR System (Applied Biosystems, USA).

Table 1 Clinical characteristics of studied patients

\begin{tabular}{ll}
\hline Age [years] & $47.55 \pm 12.96$ \\
\hline Sex [M/F] & $48 / 34$ \\
Time after transplantation [months] & $31 \pm 9$ \\
Delayed graft function & 31 patients \\
Creatinine $[\mathrm{mg} / \mathrm{dl}]$ & $1.76 \pm 0.58$ \\
Cyclosporine A [ng/ml] & $150-200$ \\
Tacrolimus $[\mathrm{ng} / \mathrm{ml}]$ & $7-12$ \\
\hline
\end{tabular}




\section{Statistical analysis}

The distribution of the genotypes and alleles in the studied sample was initially evaluated with the Hardy-Weinberg equilibrium to assess if any population deviations occurred. The frequency of the genotypes and alleles was subsequently evaluated using the Chi-squared test or Fisher's exact test. The severity of histopathological changes in renal allografts was compared between genotype groups using the nonparametric Kruskal-Wallis test followed by the Mann-Whitney test, as their distributions were significantly different from normal. $P<0.05$ without correction for multiple comparisons was considered statistically significant. The study had sufficient statistical power to detect with $80 \%$ probability differences in the severity of histopathological changes between genotype groups measured according to Banff classification on rank scale ranging from 0 to 3 (Grade $0=0$, Grade $1=1$, Grade $2=2$, Grade $3=3$ ) equal to: between 0.39 and 0.73 (depending on histopathological parameter) of degrees of Banff scale for rs2275913, 0.55-1.03 for rs2397084, $0.44-0.90$ for rs11465553 and 0.67-1.39 for rs763780. In summary, for most of the polymorphisms and histopathological parameters our study could detect the difference of a parameter between the genotype groups equivalent to one degree of Banff classification. It means that if real average value of Banff scale for the first genotype group is " 1 " corresponding "Grade 1" and for the second genotype group it is "2" corresponding to "Grade 2", then our study has enough statistical power to claim at $p<0.05$ that the histopathological changes are more severe in the second group.

\section{Results}

The distribution of all studied polymorphisms was consistent with the Hardy-Weinberg equilibrium $(P=0.59$; $P=0.51 ; P=1.0$ and $P=1.0$, respectively) and is shown in Additional file 1: Tables S1 and S2. As shown in Additional file 1: Tables S1 and S2 there were no statistically significant differences in distribution of studied polymorphisms between kidney transplant recipients in whom kidney biopsy was performed vs. kidney transplant recipients in whom no kidney biopsy was performed and between kidney transplant recipients in whom kidney biopsy was performed vs. control group.

There was a significant association between the rs2275913 IL17A gene polymorphism and the grade of tubulitis, which was more severe among renal allograft recipients with the A allele compared to patients with the GG genotype (GG vs. AG + AA, $P=0.02$ ). Arteriolar hyaline thickening was also significantly correlated with this polymorphism; however, it was more severe among patients with the $G$ allele compared to recipients with the AA genotype (AA vs. AG + GG, $P=0.01$ ). In addition, mesangial matrix increase was significantly enhanced in patients with the rs2275913 IL17A G allele compared to recipients with the AA genotype (AA vs. GG + AG, $P=$ $0.04)$. There were no statistically significant associations between the rs2275913 IL17A gene polymorphism and other histopathological changes in kidney allograft biopsies (Table 2).

As shown in Table 3, there were no significant associations between the rs2397084 IL17F gene polymorphism and histopathological changes in kidney allograft biopsies. Regarding the rs11465553 IL17F gene polymorphism, fibrous intimal thickening was significantly more severe among A allele carriers in comparison to patients with the GG genotype; however, these differences were on the border of statistical significance (GG vs. GA, $P=$ 0.086 ). There were no statistically significant associations between the rs11465553 IL17F gene polymorphism and other histopathological changes in kidney allograft biopsies (Table 4).

Table 5 shows a significant association between the rs763780 IL17F gene polymorphism and interstitial fibrosis, which was more severe among individuals with the $C$ allele than those with the TT genotype (TT vs. TC, $P=$ 0.017). Similarly, tubular atrophy was enhanced in individuals with the $\mathrm{C}$ allele than those with the TT genotype; however, these differences were on the border of statistical significance (TT vs. TC, $P=0.09$ ). There were no statistically significant associations between the rs763780 IL17F gene polymorphism and other histopathological changes in kidney allograft biopsies.

\section{Discussion}

Based on the results of previous studies, it was hypothesised that functional polymorphisms within IL-17 genes may be associated with histopathological changes in transplanted kidney biopsies. The results of this study demonstrated that the rs2275913 IL17A gene polymorphism was significantly associated with tubulitis, arteriolar hyaline thickening and mesangial matrix increase and that the rs763780 ILI7F gene polymorphism was correlated with the severity of tubular atrophy and interstitial fibrosis. However, it should be taken into account that the statistical significance was achieved without correction for multiple testing, and no significant association would remain significant after such correction. Our statistical power analysis assumed no such correction, and the numbers of patients needed to achieve the assumed power with the correction would greatly exceed logistic possibilities of our transplantation center. Therefore, these results from our exploratory study should be verified by further research.

The main reason of performing the biopsy in each case was deteriorating kidney function expressed through impaired eGFR (increase of creatinine concentration of at least $25 \%$ ) accompanied by other clinical symptoms. The 
Table 2 The associations between histopathological changes (i, t, v, ah, ci, cg, ct, cv, mm) in kidney allografts and rs2275913 IL17A gene polymorphism

\begin{tabular}{|c|c|c|c|c|c|c|c|c|c|c|c|}
\hline \multirow[t]{2}{*}{ Biopsy parameter and genotypes } & \multicolumn{2}{|c|}{$\begin{array}{l}\text { Grade } 0 \\
n \%\end{array}$} & \multicolumn{2}{|c|}{$\begin{array}{l}\text { Grade } 1 \\
n \%\end{array}$} & \multirow{2}{*}{\multicolumn{2}{|c|}{$\begin{array}{l}\text { Grade } 2 \\
n \%\end{array}$}} & \multicolumn{2}{|c|}{$\begin{array}{l}\text { Grade } 3 \\
n \%\end{array}$} & \multirow[t]{2}{*}{ Mean \pm SD } & \multicolumn{2}{|c|}{ M-W test $p$ for comparison } \\
\hline & & & & & & & & & & GGvsAG+AA & $\mathrm{AAvsAG+GG}$ \\
\hline \multicolumn{12}{|l|}{ (i) $n=72$} \\
\hline AA & 2 & 20.00 & 4 & 40.00 & 2 & 20.00 & 2 & 20.00 & $1.40 \pm 1.08$ & & \\
\hline$A G$ & 9 & 26.47 & 13 & 38.24 & 7 & 20.59 & 5 & 14.71 & $1.24 \pm 1.02$ & 0.96 & 0.68 \\
\hline GG & 9 & 32.14 & 8 & 28.57 & 5 & 17.86 & 6 & 21.43 & $1.29 \pm 1.15$ & & \\
\hline \multicolumn{12}{|l|}{ (t) $n=64$} \\
\hline $\mathrm{AA}$ & 1 & 11.11 & 4 & 44.44 & 1 & 11.11 & 3 & 33.33 & $1.67 \pm 1.12$ & & \\
\hline$A G$ & 5 & 17.24 & 13 & 44.83 & 9 & 31.03 & 2 & 6.90 & $1.28 \pm 0.84$ & 0.02 & 0.13 \\
\hline GG & 11 & 42.31 & 10 & 38.46 & 3 & 11.54 & 2 & 7.69 & $0.85 \pm 0.92$ & & \\
\hline \multicolumn{12}{|l|}{ (v) $n=57$} \\
\hline AA & 6 & 85.71 & 1 & 14.29 & 0 & 0.00 & 0 & 0.00 & $0.14 \pm 0.38$ & & \\
\hline$A G$ & 25 & 89.29 & 1 & 3.57 & 1 & 3.57 & 1 & 3.57 & $0.21 \pm 0.69$ & 0.37 & 0.64 \\
\hline GG & 21 & 95.45 & 1 & 4.55 & 0 & 0.00 & 0 & 0.00 & $0.05 \pm 0.21$ & & \\
\hline \multicolumn{12}{|l|}{ (ah) $n=68$} \\
\hline$A A$ & 6 & 66.67 & 3 & 33.33 & 0 & 0.00 & 0 & 0.00 & $0.33 \pm 0.50$ & & \\
\hline$A G$ & 8 & 25.00 & 17 & 53.13 & 7 & 21.87 & 0 & 0.00 & $0.97 \pm 0.70$ & 0.45 & 0.01 \\
\hline GG & 8 & 29.63 & 12 & 44.44 & 6 & 22.22 & 1 & 3.70 & $1.00 \pm 0.83$ & & \\
\hline \multicolumn{12}{|l|}{ (ci) $n=68$} \\
\hline AA & 6 & 60.00 & 4 & 40.00 & 0 & 0.00 & 0 & 0.00 & $0.40 \pm 0.52$ & & \\
\hline$A G$ & 16 & 50.00 & 10 & 31.25 & 5 & 15.63 & 1 & 3.13 & $0.72 \pm 0.85$ & 0.61 & 0.32 \\
\hline GG & 13 & 50.00 & 7 & 26.92 & 4 & 15.38 & 2 & 7.69 & $0.81 \pm 0.98$ & & \\
\hline \multicolumn{12}{|l|}{ (cg) $n=55$} \\
\hline AA & 7 & 100.0 & 0 & 0.00 & - & & 0 & 0.00 & $0.00 \pm 0.00$ & & \\
\hline$A G$ & 22 & 91.67 & 1 & 4.17 & - & & 1 & 4.17 & $0.17 \pm 0.64$ & 0.14 & 0.29 \\
\hline GG & 19 & 79.17 & 5 & 20.83 & - & & 0 & 0.00 & $0.21 \pm 0.42$ & & \\
\hline \multicolumn{12}{|l|}{ (ct) $n=68$} \\
\hline AA & 3 & 30.00 & 6 & 60.00 & 0 & 0.00 & 1 & 10.00 & $0.90 \pm 0.88$ & & \\
\hline$A G$ & 2 & 6.25 & 20 & 62.50 & 8 & 25.00 & 2 & 6.25 & $1.31 \pm 0.69$ & 0.97 & 0.11 \\
\hline GG & 5 & 19.23 & 13 & 50.00 & 5 & 19.23 & 3 & 11.54 & $1.23 \pm 0.91$ & & \\
\hline \multicolumn{12}{|l|}{ (cv) $n=51$} \\
\hline AA & 6 & 75.00 & 1 & 12.50 & 0 & 00.00 & 1 & 12.50 & $0.50 \pm 1.07$ & & \\
\hline$A G$ & 14 & 56.00 & 2 & 8.00 & 4 & 16.00 & 5 & 20.00 & $1.00 \pm 1.26$ & 0.79 & 0.38 \\
\hline GG & 11 & 61.11 & 3 & 16.67 & 2 & 11.11 & 2 & 11.11 & $0.72 \pm 1.07$ & & \\
\hline \multicolumn{12}{|l|}{$(\mathrm{mm}) n=60$} \\
\hline AA & 7 & 87.50 & 1 & 12.50 & 0 & 0.00 & 0 & 0.00 & $0.13 \pm 0.35$ & & \\
\hline$A G$ & 14 & 51.85 & 7 & 25.93 & 2 & 7.41 & 4 & 14.81 & $0.85 \pm 1.10$ & 0.39 & 0.04 \\
\hline GG & 12 & 48.00 & 7 & 28.00 & 3 & 12.00 & 3 & 12.00 & $0.88 \pm 1.05$ & & \\
\hline
\end{tabular}

$p$ value calculated with the Mann-Whitney's (M-W) test

$\mathrm{i}$ - interstitial infiltration, $\mathrm{t}$ - tubulitis, $\mathrm{v}$ - intimal arteritis, ah - arteriolar hyaline thickening, ci - interstitial fibrosis, cg - glomerulopathy, ct - tubular atrophy, cv fibrous intimal thickening, $\mathrm{mm}$ - mesangial matrix increase

majority of biopsy specimens were collected during the first year after transplantation because of acute rejection (AR) suspicion. In 19 cases biopsy confirmed CAD and was performed between the first and the fifth year after transplantation. Thus, taking into consideration that kidney function deterioration resulted from AR or chronic allograft dysfunction (CAD) a typical treatment was used. The treatment outcome presented as follow-up 
Table 3 The associations between histopathological changes (i, t, v, ah, ci, cg, ct, cv, mm) in kidney allografts and rs2397084 IL17F gene polymorphism

\begin{tabular}{|c|c|c|c|c|c|c|c|c|c|c|}
\hline \multirow{2}{*}{$\begin{array}{l}\text { Biopsy parameter and genotypes } \\
\text { (i) } n=72\end{array}$} & \multicolumn{2}{|c|}{$\begin{array}{l}\text { Grade } 0 \\
\text { n \% }\end{array}$} & \multicolumn{2}{|c|}{$\begin{array}{l}\text { Grade } 1 \\
\mathrm{n} \%\end{array}$} & \multicolumn{2}{|c|}{$\begin{array}{l}\text { Grade } 2 \\
\mathrm{n} \%\end{array}$} & \multicolumn{2}{|c|}{$\begin{array}{l}\text { Grade } 3 \\
\mathrm{n} \%\end{array}$} & \multirow[t]{2}{*}{ Mean \pm SD } & \multirow[t]{2}{*}{ M-W test $\mathrm{p}$ for comparison TTvsTC+CC } \\
\hline & & & & & & & & & & \\
\hline$\pi$ & 15 & 25.86 & 19 & 32.76 & 13 & 22.41 & 11 & 18.97 & $1.35 \pm 1.07$ & \\
\hline $\mathrm{TC}$ & 4 & 30.77 & 6 & 46.15 & 1 & 7.69 & 2 & 15.38 & $1.08 \pm 1.04$ & 0.26 \\
\hline CC & 1 & 100.0 & 0 & 0.00 & 0 & 0.00 & 0 & 0.00 & $0.00 \pm 0.00$ & \\
\hline \multicolumn{11}{|l|}{ (t) $n=64$} \\
\hline$\pi$ & 13 & 25.49 & 22 & 43.14 & 11 & 21.57 & 5 & 9.80 & $1.16 \pm 0.92$ & \\
\hline $\mathrm{TC}$ & 3 & 25.00 & 5 & 41.67 & 2 & 16.67 & 2 & 16.67 & $1.25 \pm 1.06$ & 0.90 \\
\hline $\mathrm{CC}$ & 1 & 100.0 & 0 & 50.00 & 0 & 0.00 & 0 & 0.00 & $0.00 \pm 0.00$ & \\
\hline \multicolumn{11}{|l|}{ (v) $n=57$} \\
\hline$\pi$ & 42 & 91.30 & 2 & 4. 35 & 1 & 2.17 & 1 & 2.17 & $0.15 \pm 0.56$ & \\
\hline $\mathrm{TC}$ & 9 & 90.00 & 1 & 10.00 & 0 & 0.00 & 0 & 0.00 & $0.10 \pm 0.32$ & 0.98 \\
\hline CC & 1 & 100.0 & 0 & 0.00 & 0 & 0.00 & 0 & 0.00 & $0.00 \pm 0.00$ & \\
\hline \multicolumn{11}{|l|}{$(a h) n=68$} \\
\hline$\pi$ & 18 & 32.14 & 26 & 46.43 & 12 & 21.43 & 0 & 0.00 & $0.89 \pm 0.73$ & \\
\hline $\mathrm{TC}$ & 4 & 36.36 & 6 & 54.55 & 1 & 9.09 & 0 & 0.00 & $0.73 \pm 0.65$ & 0.90 \\
\hline CC & 0 & 0.00 & 0 & 0.00 & 0 & 0.00 & 1 & 100.0 & $3.00 \pm 0.00$ & \\
\hline \multicolumn{11}{|l|}{ (ci) $n=68$} \\
\hline$\pi$ & 30 & 52.63 & 17 & 29.82 & 7 & 12.28 & 3 & 5.26 & $0.70 \pm 0.89$ & \\
\hline TC & 5 & 50.00 & 4 & 40.00 & 1 & 10.00 & 0 & 0.00 & $0.60 \pm 0.70$ & 0.77 \\
\hline CC & 0 & 0.00 & 0 & 0.00 & 1 & 100.0 & 0 & 0.00 & $2.00 \pm 0.00$ & \\
\hline \multicolumn{11}{|l|}{ (cg) $n=55$} \\
\hline$\pi$ & 36 & 87.70 & 4 & 9.76 & - & & 1 & 2.44 & $0.17 \pm 0.54$ & \\
\hline TC & 12 & 92.31 & 1 & 7.69 & - & & 0 & 0.00 & $0.08 \pm 0.28$ & 0.88 \\
\hline$C C$ & 0 & 0.00 & 1 & 100.0 & - & & 0 & 0.00 & $1.00 \pm 0.00$ & \\
\hline \multicolumn{11}{|l|}{ (ct) $n=68$} \\
\hline$\pi$ & 7 & 12.50 & 33 & 58.93 & 10 & 17.86 & 6 & 10.71 & $1.27 \pm 0.82$ & \\
\hline $\mathrm{TC}$ & 3 & 27.27 & 6 & 54.55 & 2 & 18.18 & 0 & 0.00 & $0.91 \pm 0.70$ & 0.38 \\
\hline$C C$ & 0 & 0.00 & 0 & 0.00 & 1 & 100.0 & 0 & 0.00 & $2.00 \pm 0.00$ & \\
\hline \multicolumn{11}{|l|}{$(c v) n=51$} \\
\hline$\pi$ & 23 & 57.50 & 5 & 12.50 & 6 & 15.00 & 6 & 15.00 & $0.88 \pm 1.16$ & \\
\hline $\mathrm{TC}$ & 7 & 70.00 & 1 & 10.00 & 0 & 0.00 & 2 & 20.00 & $0.70 \pm 1.25$ & 0.47 \\
\hline CC & 1 & 100.0 & 0 & 0.00 & 0 & 0.00 & 0 & 0.00 & $0.00 \pm 0.00$ & \\
\hline \multicolumn{11}{|l|}{$(\mathrm{mm}) \mathrm{n}=60$} \\
\hline$\pi$ & 24 & 52.17 & 13 & 28.26 & 4 & 8.70 & 5 & 10.87 & $0.78 \pm 1.01$ & \\
\hline TC & 9 & 69.23 & 2 & 15.38 & 1 & 7.69 & 1 & 7.69 & $0.54 \pm 0.97$ & 0.61 \\
\hline $\mathrm{CC}$ & 0 & 0.00 & 0 & 0.00 & 0 & 0.00 & 1 & 100.0 & $3.00 \pm 0.00$ & \\
\hline
\end{tabular}

$p$ value calculated with the Mann-Whitney's (M-W) test

$i$ interstitial infiltration, $t$ tubulitis, $v$ intimal arteritis, $a$ h arteriolar hyaline thickening, ci - interstitial fibrosis, cg - glomerulopathy, ct - tubular atrophy, cv - fibrous intimal thickening, $\mathrm{mm}$ - mesangial matrix increase

observation has been already published [14]. The main conclusion of that paper was that the GG genotype of the rs2275913 IL17A gene polymorphism was associated with significantly impaired long-term kidney allograft function, whereas the GA genotype of the rs11465553 IL17F gene polymorphism with a significantly higher risk of graft function loss and return to dialysis after kidney transplantation [14]. 
Table 4 The associations between histopathological changes (i, t, v, ah, ci, cg, ct, cv, mm) in kidney allografts and rs11465553 IL17F gene polymorphism

\begin{tabular}{|c|c|c|c|c|c|c|c|c|c|c|}
\hline $\begin{array}{l}\text { Biopsy parameter and genotypes } \\
\text { (i) } n=72\end{array}$ & \multicolumn{2}{|c|}{$\begin{array}{l}\text { Grade } 0 \\
n \%\end{array}$} & \multicolumn{2}{|c|}{$\begin{array}{l}\text { Grade } 1 \\
n \%\end{array}$} & \multicolumn{2}{|c|}{$\begin{array}{l}\text { Grade } 2 \\
n \%\end{array}$} & \multicolumn{2}{|c|}{$\begin{array}{l}\text { Grade } 3 \\
n \%\end{array}$} & Mean \pm SD & M-W test $p$ for comparison GGvsGA \\
\hline GG & 17 & 27.42 & 22 & 35.48 & 13 & 20.97 & 10 & 16.13 & $1.26 \pm 1.04$ & 0.79 \\
\hline GA & 3 & 30.00 & 3 & 30.00 & 1 & 10.00 & 3 & 30.00 & $1.40 \pm 1.27$ & \\
\hline \multicolumn{11}{|l|}{ (t) $n=64$} \\
\hline GG & 15 & 27.27 & 24 & 43.64 & 10 & 18.18 & 6 & 10.91 & $1.13 \pm 0.94$ & 0.51 \\
\hline GA & 2 & 22.22 & 3 & 33.33 & 3 & 33.33 & 1 & 11.11 & $1.33 \pm 1.00$ & \\
\hline \multicolumn{11}{|l|}{ (v) $n=57$} \\
\hline GG & 44 & 93.62 & 2 & 4.26 & 0 & 0.00 & 1 & 2.13 & $0.11 \pm 0.48$ & 0.18 \\
\hline GA & 8 & 80.0 & 1 & 10.00 & 1 & 10.00 & 0 & 0.00 & $0.30 \pm 0.68$ & \\
\hline \multicolumn{11}{|l|}{ (ah) $n=68$} \\
\hline GG & 20 & 33.90 & 27 & 45.76 & 11 & 18.64 & 1 & 1.69 & $0.88 \pm 0.77$ & 0.60 \\
\hline GA & 2 & 22.22 & 5 & 55.56 & 2 & 22.22 & 0 & 0.00 & $1.00 \pm 0.71$ & \\
\hline \multicolumn{11}{|l|}{ (ci) $n=68$} \\
\hline GG & 28 & 48.28 & 19 & 32.76 & 8 & 13.79 & 3 & 5.17 & $0.76 \pm 0.89$ & 0.21 \\
\hline GA & 7 & 70.00 & 2 & 20.00 & 1 & 10.00 & 0 & 0.00 & $0.40 \pm 0.70$ & \\
\hline \multicolumn{11}{|l|}{ (cg) $n=55$} \\
\hline GG & 40 & 85.11 & 6 & 12.77 & - & & 1 & 2.13 & $0.19 \pm 0.54$ & 0.26 \\
\hline GA & 8 & 100.0 & 0 & 0.00 & - & & 0 & 0.00 & $0.00 \pm 0.00$ & \\
\hline \multicolumn{11}{|l|}{ (ct) $n=68$} \\
\hline GG & 9 & 15.25 & 32 & 54.24 & 13 & 22.03 & 5 & 8.47 & $1.24 \pm 0.82$ & 0.56 \\
\hline GA & 1 & 11.11 & 7 & 77.78 & 0 & 0.00 & 1 & 11.11 & $1.11 \pm 0.78$ & \\
\hline \multicolumn{11}{|l|}{ (cv) $n=51$} \\
\hline GG & 29 & 64.44 & 6 & 13.33 & 4 & 18.89 & 6 & 13.33 & $0.71 \pm 1.10$ & 0.086 \\
\hline GA & 2 & 33.33 & 0 & 0.00 & 2 & 33.33 & 2 & 33.33 & $1.67 \pm 1.37$ & \\
\hline \multicolumn{11}{|l|}{$(\mathrm{mm}) n=60$} \\
\hline GG & 28 & 52.83 & 14 & 26.42 & 4 & 7.55 & 7 & 13.21 & $0.81 \pm 1.06$ & 0.36 \\
\hline GA & 5 & 71.43 & 1 & 14.29 & 1 & 14.29 & 0 & 0.00 & $0.43 \pm 0.79$ & \\
\hline
\end{tabular}

$p$ value calculated with the Mann-Whitney's $(M-W)$ test

$i$ interstitial infiltration, $t$ tubulitis, $v$ intimal arteritis, $a h$ arteriolar hyaline thickening, $c i$ interstitial fibrosis, $c g$ glomerulopathy, $c t$ tubular atrophy, $c v$ fibrous intimal thickening, $\mathrm{mm}$ mesangial matrix increase

A biopsy is the gold standard for diagnosing kidney allograft disorders as it allows the verification of the probable causes of organ function deterioration. Therefore biopsies are performed in kidney recipients with DGF or suspected AR to assess the extent of chronic rejection [15]. The Banff working classification criteria are currently used to assess renal transplant pathology and facilitate the selection of appropriate treatments [16]. Histopathological changes in transplanted kidneys are associated with many immunological and nonimmunological factors; for example, ischemia-reperfusion injury not only damages the organ in the early posttransplant period but also triggers chronic inflammation leading to progressive interstitial fibrosis [17]. Glomerulopathy, tubular atrophy and interstitial fibrosis are the main morphological causes of CAD.
Interleukins are key mediators of the alloresponse, and the role of IL-17 in kidney transplantation has yet to be elucidated $[18,19]$. IL-17 is present in different types of rejection, as it can affect both the innate and acquired immune response through neutrophil activation and by acting on antigen-presenting cells. One important function of IL-17 is inducing the production of cytokines and chemokines, including IL-1 $\beta$, IL-6, IL- 8 and tumour necrosis factor- $\alpha$ (TNF- $\alpha)$ [20], therefore there is growing interest in the various functions of IL-17 in transplantation research. Loverre et al. showed that transplanted kidneys undergoing DGF and AR were characterised by significantly higher levels of IL-17(+) cells. Moreover, the number of IL-17(+) cells was significantly increased in grafts with T-cell mediated AR in 
Table 5 The associations between histopathological changes (i, t, v, ah, ci, cg, ct, cV, mm) in kidney allografts and rs763780 IL17F gene polymorphism

\begin{tabular}{|c|c|c|c|c|c|c|c|c|c|c|}
\hline \multirow{2}{*}{$\begin{array}{l}\text { Biopsy parameter and genotypes } \\
\text { (i) } n=72\end{array}$} & \multicolumn{2}{|c|}{$\begin{array}{l}\text { Grade } 0 \\
n \%\end{array}$} & \multicolumn{2}{|c|}{$\begin{array}{l}\text { Grade } 1 \\
n \%\end{array}$} & \multicolumn{2}{|c|}{$\begin{array}{l}\text { Grade } 2 \\
n \%\end{array}$} & \multicolumn{2}{|c|}{$\begin{array}{l}\text { Grade } 3 \\
n \%\end{array}$} & \multirow[t]{2}{*}{ Mean \pm SD } & \multirow[t]{2}{*}{ M-W test $p$ for comparison TVsTC } \\
\hline & & & & & & & & & & \\
\hline$\pi$ & 20 & 29.85 & 22 & 32.84 & 13 & 19.40 & 12 & 17.91 & $1.25 \pm 1.08$ & 0.43 \\
\hline $\mathrm{TC}$ & 0 & 0.00 & 3 & 60.00 & 1 & 20.00 & 1 & 20.00 & $1.60 \pm 0.89$ & \\
\hline \multicolumn{11}{|l|}{ (t) $n=64$} \\
\hline$\pi$ & 17 & 27.87 & 24 & 39.34 & 13 & 21.31 & 7 & 11.48 & $1.16 \pm 0.97$ & 0.89 \\
\hline TC & 0 & 0.00 & 3 & 100.0 & 0 & 0.00 & 0 & 0.00 & $1.00 \pm 0.00$ & \\
\hline \multicolumn{11}{|l|}{ (v) $n=57$} \\
\hline$\pi$ & 47 & 90.38 & 3 & 5.77 & 1 & 1.92 & 1 & 1.92 & $0.15 \pm 0.54$ & 0.49 \\
\hline TC & 5 & 100.0 & 0 & 0.00 & 0 & 0.00 & 0 & 0.00 & $0.00 \pm 0.00$ & \\
\hline \multicolumn{11}{|l|}{ (ah) $n=68$} \\
\hline$\pi$ & 21 & 33.33 & 30 & 47.62 & 11 & 17.46 & 1 & 1.59 & $0.87 \pm 0.75$ & 0.34 \\
\hline TC & 1 & 20.00 & 2 & 40.00 & 2 & 40.00 & 0 & 0.00 & $1.20 \pm 0.84$ & \\
\hline \multicolumn{11}{|l|}{ (ci) $n=68$} \\
\hline$\pi$ & 35 & 55.56 & 18 & 28.57 & 8 & 12.70 & 2 & 3.17 & $0.64 \pm 0.83$ & 0.017 \\
\hline TC & 0 & 0.00 & 3 & 60.00 & 1 & 20.00 & 1 & 20.00 & $1.60 \pm 0.89$ & \\
\hline \multicolumn{11}{|l|}{ (cg) $n=55$} \\
\hline$\pi$ & 44 & 88.00 & 5 & 10.00 & - & & 1 & 2.00 & $0.16 \pm 0.51$ & 0.65 \\
\hline TC & 4 & 80.00 & 1 & 20.00 & - & & 0 & 0.00 & $0.20 \pm 0.45$ & \\
\hline \multicolumn{11}{|l|}{ (ct) $n=68$} \\
\hline$\pi$ & 10 & 15.87 & 37 & 58.73 & 11 & 17.46 & 5 & 7.94 & $1.18 \pm 0.79$ & 0.09 \\
\hline $\mathrm{TC}$ & 0 & 0.00 & 2 & 40.00 & 2 & 40.00 & 1 & 20.00 & $1.80 \pm 0.84$ & \\
\hline \multicolumn{11}{|l|}{ (cv) $n=51$} \\
\hline$\pi$ & 28 & 60.42 & 6 & 12.50 & 6 & 12.50 & 7 & 14.58 & $0.83 \pm 1.15$ & 0.75 \\
\hline TC & 3 & 75.00 & 0 & 0.00 & 0 & 0.00 & 1 & 25.00 & $0.75 \pm 1.50$ & \\
\hline \multicolumn{11}{|l|}{$(\mathrm{mm}) n=60$} \\
\hline$\pi$ & 30 & 54.55 & 15 & 27.27 & 5 & 9.09 & 5 & 9.09 & $0.73 \pm 0.97$ & 0.72 \\
\hline $\mathrm{TC}$ & 3 & 60.00 & 0 & 0.00 & 0 & 0.00 & 2 & 40.00 & $1.20 \pm 1.64$ & \\
\hline
\end{tabular}

$\mathrm{p}$ value calculated with the Mann-Whitney's (M-W) test

$\mathrm{i}$ - interstitial infiltration, $\mathrm{t}$ - tubulitis, $\mathrm{v}$ - intimal arteritis, ah - arteriolar hyaline thickening, ci - interstitial fibrosis, cg - glomerulopathy, ct - tubular atrophy, cv fibrous intimal thickening, $\mathrm{mm}$ - mesangial matrix increase

comparison to grafts with morphological symptoms of antibody-mediated rejection, interstitial fibrosis, tubular atrophy and acute tubular damage due to calcineurin-inhibitor toxicity. Nevertheless, IL-17(+) cells were observed in all studied allografts [21, 22]. Previous studies showed that the $\mathrm{G}$ allele of the rs2275913 IL17A gene polymorphism was significantly correlated with a higher risk of DGF and higher long-term creatinine concentrations [14]. DGF occurs as a result of ischemia-reperfusion injury, and the outcomes of these complications include chronic lesions of the allograft which are manifested through tubular atrophy, interstitial fibrosis and mesangial matrix increase. Besides the histopathological changes, a decrease in allograft function can also be observed [23]. In this study, the G allele of the rs2275913
IL17A gene polymorphism was associated with a significantly enhanced mesangial matrix increase. This result is assumed to be a logical causal link with the earlier findings. Similarly, the $\mathrm{C}$ allele of the rs763780 IL17F gene polymorphism was correlated with higher long-term creatinine concentrations in previous studies, and this study reported its association with the severity of tubular atrophy and interstitial fibrosis.

Research regarding hepatitis B virus infection in kidney transplant and bone marrow recipients revealed that the A allele of the rs2275913 IL17A gene polymorphism was implicated in AR and graft-versus-host disease [24, 25]. In this study, the A allele of this polymorphism was significantly associated with tubulitis, which is the hallmark manifestation of the acute immune response. 
Arteriolar hyaline thickening, which is a type of arteriolosclerosis, is another histopathological lesion that is associated with ageing, hypertension, diabetes mellitus and, in the case of kidney transplant recipients, calcineurin inhibitor toxicity. Arteriolar hyalinosis is often found in kidney pathology because of its multifactorial background [26]. In this study, the severity of arteriolar hyaline thickening may be related to the mesangial matrix increase due to the association of these lesions with the G allele of the rs2275913 IL17A gene polymorphism; however, it may also be an incidental or calcineurin inhibitor toxicity-related result that requires further research.

Animal studies revealed that IL-17 was implicated in chronic rejection. A decreased immune response leading to prolonged allograft survival after heart transplantation was observed in IL-17 deficient mice [27]. Moreover, it was shown that Tregs were responsible for downregulation of Th17 and thus lowering IL-17 levels during chronic inflammation [28]. Recent studies in a murine model of kidney allograft confirmed that IL-17 deficiency or neutralisation was protective against rejection and, more importantly, that this process may be mediated by Tregs [29]. Unfortunately, studies regarding the role of IL-17 gene polymorphisms in chronic rejection in humans are lacking, and this makes comparing the obtained results rather difficult.

\section{Conclusions}

The results of this study may suggest a possible association between the rs2275913 IL17A and rs2275913 IL17A gene polymorphisms and some histopathological changes in transplanted kidney biopsies. However, further studies are needed to get more insight into the functional relevance of the polymorphisms examined with respect to long term kidney allograft outcome.

\section{Additional file}

Additional file 1: Table S1. The distribution of $I L 17 A$ and $I L 17 F$ genotypes in control group vs. kidney transplant recipients in whom kidney biopsy was performed. Table S2. The distribution of ILI7A and ILI7F genotypes in kidney transplant recipients in whom kidney biopsy was performed vs. kidney transplant recipients in whom no kidney biopsy was performed. (DOCX $22 \mathrm{~kb}$ )

\section{Acknowledgements}

Not applicable.

\section{Funding}

Pomeranian Medical University in Szczecin, Poland.

The financing institution did not have any role in the design of the study and collection, analysis, and interpretation of data and in writing the manuscript.

\section{Availability of data and materials}

The electronic file containing data used to support the findings of this study is available from the corresponding author upon request.

\section{Authors' contributions}

LD -study design, patients collection, data analysis; KK-manuscript preparation, data analysis, MP- patient collection, data analysis; MW-patient collection, data analysis, KS - statistical analysis, JS- patient collection, data analysis, TS- patient collection, data analysis, MS-genetic analysis, AP -study design, manuscript preparation. All authors have read and approved the manuscript.

\section{Ethics approval and consent to participate}

All procedures performed in studies involving human participants were in accordance with the ethical standards of the institutional and/or national research committee and with the 1964 Helsinki declaration and its later amendments or comparable ethical standards.

The local ethics committee at the Pomeranian Medical University, Szczecin, Poland, approved the study protocol (BN 35/2000) and written informed consent was obtained from all individual participants included in the study.

Consent for publication

Not applicable.

\section{Competing interests}

The authors declare that they have no conflict of interests.

\section{Publisher's Note}

Springer Nature remains neutral with regard to jurisdictional claims in published maps and institutional affiliations.

\section{Author details}

'Department of Nephrology, Transplantology and Internal Medicine, Pomeranian Medical University in Szczecin, Szczecin, Poland. ${ }^{2}$ Department of Surgery, University Medical Center Greifswald, Greifswald, Germany.

${ }^{3}$ Department of Biochemistry and Medical Chemistry, Pomeranian Medical University in Szczecin, Szczecin, Poland. ${ }^{4}$ Department of Surgery, Pomeranian Medical University in Szczecin, Szczecin, Poland. ${ }^{5}$ Department of Physiology, Pomeranian Medical University in Szczecin, Powstancow Wlkp. 72, 70-111 Szczecin, Poland.

Received: 13 September 2018 Accepted: 21 March 2019

Published online: 08 April 2019

\section{References}

1. Jin W, Dong C. IL-17 cytokines in immunity and inflammation. Emerg Microbes Infect. 2013;2:e60.

2. Yang X, Chang SH, Park H, Nurieva R, Shah B, Acero L, et al. Regulation of inflammatory responses by IL-17F. J Exp Med. 2008;205:1063-75.

3. Isailovic N, Daigo K, Mantovani A, Selmi C. Interleukin-17 and innate immunity in infections and chronic inflammation. J Autoimmun. 2015; 60:1-11.

4. Croxford AL, Mair F, Becher B. IL-23: one cytokine in control of autoimmunity. Eur J Immunol. 2012;42:2263-73.

5. Ito H, Yamada H, Shibata TN, Mitomi H, Nomoto S, Ozaki S, et al. Dual role of interleukin-17 in pannus growth and osteoclastogenesis in rheumatoid arthritis. Arthritis Res Ther. 2011;13:R14.

6. Singh B, Schwartz JA, Sandrock C, Bellemore SM, Nikoopour E. Modulation of autoimmune diseases by interleukin (IL)-17 producing regulatory $T$ helper (Th17) cells. Indian J Med Res. 2013;138:591-4.

7. Akalin E, Murphy B. Gene polymorphisms and transplantation. Curr Opin Immunol. 2001;13:572-6.

8. Wang X, Zhang Y, Yang XO, Nurieva Rl, Chang SH, Ojeda SS, et al. Transcription of IL 17 and IL $17 f$ is controlled by conserved noncoding sequence 2. Immunity. 2012;36:23-31.

9. Konya C, Paz Z, Apostolidis SA, Tsokos GC. Update on the role of interleukin 17 in rheumatologic autoimmune diseases. Cytokine. 2015;75:207-15.

10. Karimi MH, Hejr S, Geramizadeh B, Yaghobi R, Sagheb MM, KamaliSarvestani $E$, et al. Combined analysis of cytokine gene polymorphism and the level of expression with allograft function in kidney transplant recipients. Transpl Immunol. 2014;30:46-51.

11. Yapici Ü, Kers J, Bemelman FJ, Roelofs JJ, Groothoff JW, van der Loos CM, et al. Interleukin-17 positive cells accumulate in renal allografts during acute rejection and are independent predictors of worse graft outcome. Transpl Int. 2011;24:1008-17. 
12. Millán O, Rafael-Valdivia L, San Segundo D, Boix F, Castro-Panete MJ, LópezHoyos M, et al. Should IFN- $\gamma$, IL-17 and IL-2 be considered predictive biomarkers of acute rejection in liver and kidney transplant? Results of a multicentric study. Clin Immunol. 2014;154:141-54.

13. Park H, Shin S, Park MH, Kim YS, Ahn C, Ha J, et al. Association of IL-17F gene polymorphisms with renal transplantation outcome. Transplant Proc. 2014;46:121-3.

14. Romanowski M, Kłoda K, Osękowska B, Domański L, Pawlik A, Safranow K, et al. Influence of the IL17A and IL17F gene polymorphisms on the long-term kidney allograft function and return to dialysis after kidney transplantation. Clin Transpl. 2015:29:1187-94.

15. Domanski L, Kłoda K, Pawlik A, Wisniewska M, Kwiatkowska E, Kurzawski M, et al. Correlation between ICAM1 and VCAM1 gene polymorphisms and histopathological changes in kidney allograft biopsies. Arch Med Sci. 2013;9: 276-82.

16. Solez K. History of the Banff classification of allograft pathology as it approaches its 20th year. Curr Opin Organ Transplant. 2010;15:49-51.

17. Kwiatkowska E, Domański L, Bober J, Kłoda K, Safranow K, SzymańskaPasternak J, et al. N-acetyl-beta-glucosaminidase urine activity as a marker of early proximal tubule damage and a predictor of the long-term function of the transplanted kidneys. Acta Biochim Pol. 2014;61:275-80.

18. Afzali B, Lechler Rl, Hernandez-Fuentes MP. Allorecognition and the alloresponse: clinical implications. Tissue Antigens. 2007;69:545-56.

19. Afzali B, Lombardi G, Lechler RI, Lord GM. The role of Thelper 17 (Th17) and regulatory T cells (Treg) in human organ transplantation and autoimmune disease. Clin Exp Immunol. 2007;148:32-46.

20. Iwakura $Y$, Ishigame H, Saijo S, Nakae S. Functional specialization of interleukin-17 family members. Immunity. 2011;34:149-62.

21. Loverre A, Divella C, Castellano G, Tataranni T, Zaza G, Rossini M, et al. T helper 1, 2 and 17 cell subsets in renal transplant patients with delayed graft function. Transpl Int. 2011;24:233-42.

22. Loverre A, Tataranni T, Castellano G, Divella C, Battaglia M, Ditonno P, et al. IL-17 expression by tubular epithelial cells in renal transplant recipients with acute antibody-mediated rejection. Am J Transplant. 2011;11:1248-59.

23. Burne-Taney MJ, Yokota N, Rabb H. Persistent renal and extrarenal immune changes after severe ischemic injury. Kidney Int. 2005;67:1002-9.

24. Espinoza JL, Takami A, Nakata K, Onizuka M, Kawase T, Akiyama H, et al. A genetic variant in the IL-17 promoter is functionally associated with acute graft-versus-host disease after unrelated bone marrow transplantation. PLoS One. 2011;6:e26229.

25. Hejr S, Karimi MH, Yaghobi R, Kamali-Sarvestani E, Geramizadeh B, Roozbeh J. Association of IL-17, IL-21, and IL-23R gene polymorphisms with HBV infection in kidney transplant patients. Viral Immunol. 2013;26:201-6.

26. Tanabe T, Morita K, Fujita H, Hatanaka K, Ogawa Y, Hirose T, et al. Long-term clinicopathological impact of calcineurin inhibitor cessation without specific cytoreductive induction in kidney transplantation. Clin Transpl. 2013;26:9-13.

27. Itoh S, Nakae S, Axtell RC, Velotta JB, Kimura N, Kajiwara N, et al. IL-17 contributes to the development of chronic rejection in a murine heart transplant model. J Clin Immunol. 2010;30:235-40.

28. Lei J, He F, Wu M, Zheng X, Chen X, Chen Z. Administration of antiinterleukin-6 monoclonal antibody prolongs cardiac allograft survival. Transpl Int. 2010;23:1271-81.

29. Kwan T, Chadban SJ, Ma J, Bao S, Alexander SI, Wu H. IL-17 deficiency attenuates allograft injury and prolongs survival in a murine model of fully MHC-mismatched renal allograft transplantation. Am J Transplant. 2015;15: $1555-67$.

Ready to submit your research? Choose BMC and benefit from:

- fast, convenient online submission

- thorough peer review by experienced researchers in your field

- rapid publication on acceptance

- support for research data, including large and complex data types

- gold Open Access which fosters wider collaboration and increased citations

- maximum visibility for your research: over $100 \mathrm{M}$ website views per year

At BMC, research is always in progress.

Learn more biomedcentral.com/submissions 